
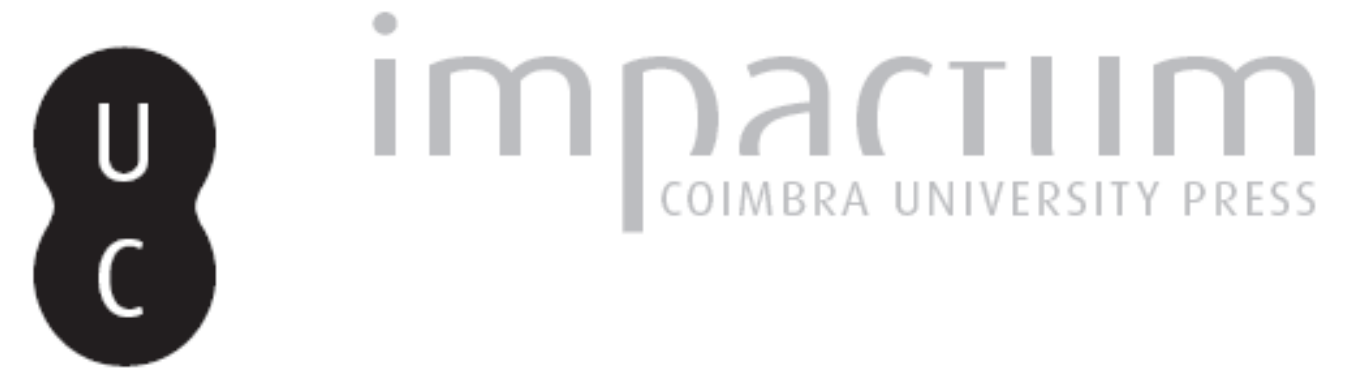

\title{
Propuesta de una guía para la valoración médico-legal de la alteración estética: daño estetico/deformidad
}

Autor(es): $\quad$ Bouchardet, $\mathrm{FCH}$; del Río, MT Criado del

Publicado por: Imprensa da Universidade de Coimbra

URL persistente:

URI:http://hdl.handle.net/10316.2/33212

DOI:

DOI:http://dx.doi.org/10.14195/1647-8630_21_6

Accessed : $\quad$ 26-Apr-2023 10:42:43

A navegação consulta e descarregamento dos títulos inseridos nas Bibliotecas Digitais UC Digitalis, UC Pombalina e UC Impactum, pressupõem a aceitação plena e sem reservas dos Termos e Condições de Uso destas Bibliotecas Digitais, disponíveis em https://digitalis.uc.pt/pt-pt/termos.

Conforme exposto nos referidos Termos e Condições de Uso, o descarregamento de títulos de acesso restrito requer uma licença válida de autorização devendo o utilizador aceder ao(s) documento(s) a partir de um endereço de IP da instituição detentora da supramencionada licença.

Ao utilizador é apenas permitido o descarregamento para uso pessoal, pelo que o emprego do(s) título(s) descarregado(s) para outro fim, designadamente comercial, carece de autorização do respetivo autor ou editor da obra.

Na medida em que todas as obras da UC Digitalis se encontram protegidas pelo Código do Direito de Autor e Direitos Conexos e demais legislação aplicável, toda a cópia, parcial ou total, deste documento, nos casos em que é legalmente admitida, deverá conter ou fazer-se acompanhar por este aviso.

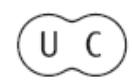




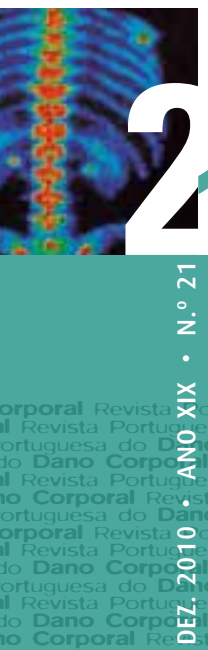

REVISTA PORTUGUESA

do

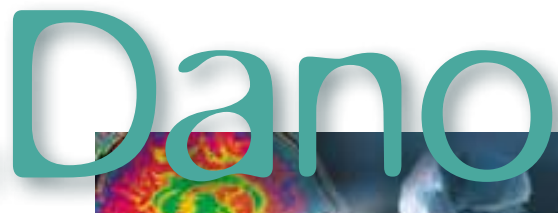

$\dot{z}$

$\dot{x}$

$\frac{x}{x}$

o. corporal Corporal Revista Portuguesa do Dano Corporal

Z

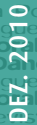

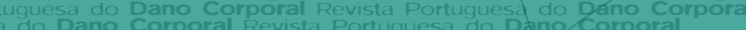

Revista Portuguesa do Dano Corporal Revista Rortuguesa do Dano Corporal

Sa do Dano Corporal Revista Portuguesa do Dano Corporal Revista Portuguesł do Dano Corporal

Portuguesa do Dano Corporal Revista portuguesta do Dano Corporal 


\title{
Propuesta de una guía para la valoración médico-legal de la alteración estética: daño estético/deformidad
}

\author{
FCH Bouchardet ${ }^{1}$, MT Criado del Río²
}

\section{Conceptos}

El daño estético es toda alteración de la morfología externa o alteración funcional corporal de la persona que se traduce en una pérdida o disminución de la belleza o atracción respecto a su estado anterior. Se trata de toda disminución o pérdida de la belleza o atracción del lesionado (CRIADO DEL RÍO, 2010). Si decimos que una persona es bella o "estética" no nos apoyamos en conceptos determinados, sino en criterios subjetivos que determinan los sentimientos de agrado o desagrado que nos genera la persona.

Algunos autores a definen como "qualquer irregularidade física ou alteração corporal externa, visível e permanente que pressuponha fealdade ostensiva à simples vista" (VIEIRA E QUINTERO, 2008).

Aunque habitualmente la palabra deformidad nos conduce a pensar casi exclusivamente en las cicatrices, debe recordarse que deformidad es un concepto mucho más amplio porque se refiere a la afectación del derecho a la propia imagen de la persona y a la pérdida de su patrimonio estético entendido como la alteración de la imagen que podemos percibir por cualquier sentido (vista, oído, olfato, tato o gusto) (COBO, 2004).

En el Derecho, la pérdida de atracción causada por una lesión viene reflejada por los términos perjuicio o daño estético y deformidad. Las repercusiones

1 Doutoranda em Ciências da Saúde e Mestre em Medicina Legal e Ciências Forenses pela Universidade de Coimbra; Especialista em Odontologia Legal pela USP - Brasil

2 Profesora Titular de Medicina Legal y Toxicología de la Faculdad de Medicina de la Universidad de Zaragoza - Espanha; Especialista en Medicina Legal y Forense; Máster universitario de valoración del daño corporal; Coordinadora del Máster de Valoración médico legal a la persona y medicina del seguro de la Universidad de Zaragoza 
de una alteración estética sobre la persona son siempre las mismas, pero la concepción de daño estético en el Derecho civil, difiere de la que se tiene de la deformidad en el Derecho penal.

\section{Aspectos civiles del perjuicio estético}

El daño estético es un daño extrapatrimonial por el que entendemos la "disminución o pérdida de la atracción o belleza del lesionado debida a las alteraciones anatómicas o funcionales causadas por el hecho lesivo del que hay de responder civilmente." Se trata de la pérdida de atracción del lesionado respecto a su estado anterior (CRIADO DEL RÍO, 2010). "El perjuicio estético consiste en cualquier modificación peyorativa que afecta a la imagen de la persona; constituye una dimensión diversa del perjuicio fisiológico que le sirve de sustrato; y refiere tanto a su expresión estática como dinámica" (COBO, 2010).

El problema principal de este daño cuya existencia se objetiva por los sentidos, principalmente la vista, reside en su cuantificación o en la medición de su importancia o gravedad, porque el grado de fealdad adquirido tras un hecho lesivo generador de responsabilidad civil es de apreciación subjetiva por el que lo padece, por el que lo valora (médico, odontólogo) y por el que lo repara (entidad aseguradora, abogado, juez).

La valoración médico legal de este daño se realiza utilizando el método descriptivo y, una vez descritas todas sus características con las que obtenemos todos los parámetros o criterios en los que nos apoyamos para comprender su dimensión, se utilizan métodos con los que se intenta establecer la entidad del daño de forma justa y equitativa con la finalidad de realizar valoraciones homogéneas y que los lesionados con un daño estético similar perciban una indemnización semejante, para eliminar el problema de la discrepancia de las valoraciones médico legales y las reparaciones jurídicas, y por lo tanto, de las injusticias, inseguridad jurídica y litigiosidad.

El método de valoración médico legal de cuantificación de este daño que se utiliza en la mayor parte de los países europeos semejantes por su doctrina jurídica y médico legal es el cualitativo. Este método que España y Portugal lo tienen incluido oficialmente en la norma legal, consiste de establecer la importancia o intensidad de este daño una vez descrito equiparándolo a una de las categorías o adjetivos referidos en una escala gradual calificativa, de 6 o 7 grados de gravedad (P. ej. la tabla española civil oficial para los accidentes de tráfico del RD 8/2004 incluye estos 6 grados: ligero, medio, moderado e importante, bastante importante y muy importante). A cada uno de los grados de la escala calificativa se le hace corresponder una cantidad económica (p. 
ej. Francia y Portugal) ó puntos que después se traducen en euros (p. ej. España). Además, se han desarrollado métodos cuantitativos con la finalidad de obtener una cifra que después se pueda trasladar a la escala calificativa de forma proporcional.

A diferencia de la mayor parte de los países europeos, en Brasil solo se utiliza el método descriptivo. Se describen las repercusiones de la secuela en una perspectiva estática y dinámica. La edad, sexo, factores sociales/escolares, familiares,... son aspectos que dependen del perito que decide incluirlos o nó en su evaluación del daño estético. No existen parámetros ni métodos específicos para la valoración del daño estético.

\section{Aspectos penales del perjuicio estético}

La deformidad citada en los diversos Códigos Penales es un término equivalente al perjuicio estético en Derecho Civil. En ambos campos del derecho el punto de partida común es la alteración estética causada por una alteración anatómica o funcional. Sin embargo, como antes decíamos, existe un matiz diferenciador fundamental entre los dos. Mientras en el Derecho civil únicamente se tiene en cuenta en la valoración médico legal y reparación judicial de este daño, la pérdida de atracción causada por la alteración estética y se prescinde de la valoración de las consecuencias que tiene la pérdida de atracción en los diferentes ámbitos de la vida de la persona (formación, laboral, social y del tiempo libre, y afectivo-familiar) porque las diferentes incapacidades laboral, de formación,... son daños independientes entre si y también del daño estético. En el derecho penal se tiene en cuenta no solo la alteración estética si no también las repercusiones que ésta tiene en las actividades de la vida diaria del lesionado para estimar su gravedad.

En España el Código penal diferencia el delito de lesiones (art. 147.1 del $\mathrm{CP}$ ) del delito de lesiones agravado en donde se encuentran los términos de deformidad (art. 150 CP) y de grave deformidad (art. 149 CP). La deformidad ha sido definida por la jurisprudencia como "toda alteración de la forma, permanente y visible”. Prescindiendo de la interpretación jurídica del concepto de deformidad, el problema se plantea en la práctica con esta cuestión ¿Toda alteración estética da lugar a una deformidad? ¿Una simple cicatriz en cualquier parte del cuerpo es siempre constitutiva de una deformidad? ¿Cuáles son los criterios que diferencian la alteración estética que implica tan solo un delito de lesiones, de un delito de lesiones agravado por una deformidad o por una grave deformidad que conlleva una mayor pena? Es el juez el que califica la conducta delictiva de lesiones, su grado y agravantes, pero la valoración médico legal de la lesión que produce una alteración estética la 
debe proporcionar el experto en valoración médico legal de daños personales de tal forma que aporte todos los elementos de la lesión necesarios para que la justicia la califique correctamente.

Debemos diferenciar la alteración anatómica o funcional que solo causa una alteración estética, de la alteración estética que causa una alteración de la atracción del lesionado y que no repercute en las actividades de la vida diaria de la persona, de la alteración estética que causa una alteración de la atracción y además incide negativamente en las actividades de la vida diaria produciendo algún tipo de incapacidad laboral, vida afectivo familiar.... Con lo expuesto puede parecer sencillo establecer la diferenciación entre el delito de lesiones, la deformidad y la grave deformidad puesto que las tres situaciones descritas se pueden hacer corresponder con los tres tipos conductas delictivas de lesiones. Así como, la grave deformidad se puede diferenciar de la deformidad en el momento que la pérdida de atracción causa una incapacidad o alteración en el desarrollo de las actividades de la vida diaria del lesionado y de sus relaciones interpersonales, la diferenciación entre la alteración estética que causa un delito de lesiones no agravado de un delito de lesiones agravado por la deformidad no lo es tanto. Su distinción estriba en la producción o no de una pérdida de atracción por la alteración estética y... ¿Cuándo una cicatriz produce una pérdida de atracción o cuando no la produce? ¿Dónde está el límite? Nuevamente nos movemos dentro de parámetros subjetivos que dependen de la libre apreciación y criterios de quienes tienen que valorar y calificar esta situación.

Aunque los CP brasileño y español difieran entre si, plantean problemas semejantes. El Código penal brasileño implica el deber de realizar la distinción entre la alteración estética constitutiva de una deformidad de la que no produce una deformidad permanente. El párrafo $\$ 2^{\circ}$ del artículo $129^{3}$ del Código Penal Brasileño se refiere únicamente a la deformidad permanente. De acuerdo con Genival Veloso França (2001) se define deformidad como toda alteración estética grave capaz de reducir, más o menos acentuadamente, la estética individual. Es la pérdida del aspecto habitual. Como afirma Almeida Jr.

3 Artigo 129 - caput - Ofender a integridade corporal ou a saúde de outrem: Pena - Detenção, 3 meses a 1 ano.

Lesão corporal de natureza grave $-\S 1^{\circ}$ Se resulta: I - Incapacidade para as ocupações habituais, por mais de 30 (trinta) dias.; II - Perigo de vida; III - Debilidade permanente do membro, sentido ou função.; IV - Aceleração do parto. Pena - Reclusão, 1 a 5 anos.

Lesão corporal de natureza gravíssima - $\S 2^{\circ}$ Se resulta: I - Incapacidade permanente para o trabalho.; II - Enfermidade incurável.; III - Perda ou inutilização de membro, sentido ou função.; IV - Deformidade permanente.; V - Aborto. Pena - Reclusão, 2 a 8 anos.

Lesão corporal seguida de morte $-\S 3^{\circ}$ Se resulta morte e as circunstâncias evidenciam que o agente não quis o resultado, nem assumiu o risco de produzi-lo: Pena - Reclusão, 4 a 12 anos 
y Costa (1978) y Fávero, (1975) el daño estético debe ser con cierta intensidad y que debe causar una incomodidad permanente, un vejamen constante para el ofendido, pero sin alcanzar los límites de cosa horripilante, de la persona lesionada.

No se ha desarrollado una valoración normalizada ni un método pericial médico para valoración penal de la alteración estética. Desde la perspectiva médico legal, Cobo Plana, J. A. (2004) y Aso, J., y Cobo, J. A. (2006) con la finalidad de aportar criterios proponen utilizar para la valoración de la intensidad de la alteración estética en el Derecho penal, el método cualitativo que se utiliza en el Derecho civil, de tal forma que consideran que el grado ligero de la escala se podría hacer corresponder con el delito de lesiones, los grados medio, moderado e importante con la deformidad, y los grados bastante o muy importante del capítulo especial de la tabla VI del Anexo del RD legislativo 8/2004 se podrían hacer corresponder con la grave deformidad.

Desde nuestra perspectiva basta con una incapacidad para una de las actividades de la vida diaria para establecer la diferencia entre deformidad y grave deformidad, pero la propuesta de estos autores podría ser adecuada para diferenciar con un criterio normalizado el delito de lesiones de una deformidad y garantizar cierta congruencia entre los dos ámbitos, diferentes en su generación de responsabilidad, pero similares en el objetivo de medir la gravedad del daño estético provocado.

\section{Finalidad de la guía para la valoración médico-legal de la alteración esté- tica: daño estético/deformidad}

Es necesario que los profesionales vinculados con la valoración y reparación del daño estético, dominen los aspectos particulares de los informes periciales en cada uno de los ámbitos del Derecho, teniendo presente su finalidad y todos los aspectos de la alteración estética con repercusión médico legal o jurídica, sin olvidar la trascendencia del alcance de sus conclusiones.

Sin embargo, la elaboración de los informes periciales se fundamenta en el estudio clínico completo del lesionado, de sus lesiones y secuelas, así como de todas sus consecuencias en los diferentes ámbitos de la persona, cuya metodología es invariable de unos casos a otros. Es después una vez recogidos todos los datos clínicos y con repercusión médico legal de las lesiones, en nuestro caso de la lesión que causa una alteración estética, cuando se procede a la elaboración de las consideraciones y concusiones médico legales que contendrán los matices particulares de cada ámbito del derecho.

En el caso del daño estético / deformidad permanente se producen los mismos problemas que con los demás daños personales. Médico-legalmente 
y jurídicamente necesitamos armonizar los criterios sobre su concepto, delimitación de su existencia e inexistencia, sus características, sus métodos de valoración y, los métodos de reparación para la indemnización civil y de calificación penal de las lesiones con una deformidad. Son muchos los intentos que se han llevado a cabo internacionalmente para la homogenización de los criterios de valoración médico legal de los diferentes daños personales, pero la citada armonización aún no se ha conseguido. La tarea es difícil, sobre todo cuando hablamos de daños de apreciación subjetiva.

Dar soluciones para obtener una valoración justa y equitativa es muy difícil. Tomando como referencia los criterios y métodos de la valoración del daño estético, consideramos que aportamos en la propuesta de la presente Guía para la valoración médico legal de la alteración estética todas las herramientas necesarias para valorar la impresión de la pérdida de atracción sin la interferencia de factores externos subjetivos, a fin de facilitar no solo la práctica de la valoración médico legal del daño, sino también la judicial, al incorporar los elementos médico legales necesarios para establecer la reparación/compensación económica en el derecho civil o calificar la lesión o grave deformidad en el Derecho penal.

Con la finalidad de contribuir en la creación de unas bases comunes, la propuesta de esta "Guía para la valoración médico-legal de la alteración estética" que presentamos facilita la obtención no solo de los datos clínicos de la alteración estética, sino también de los criterios necesarios para la aplicación de los diferentes métodos cualitativos y cuantitativos (analíticos y matemáticos) de valoración médico legal del daño estético. Métodos de valoración médico legal que posteriormente los jueces pueden utilizar para cuantificar la entidad del daño estético en derecho civil y para delimitar la diferencia entre la conducta delictiva de lesiones de la grave deformidad en derecho penal.

Finalmente, nuestro objetivo no es otro que aportar una guía, cuya aplicación y desarrollo, solo la demostrará la experiencia médico legal y jurídica de todos los interesados en la valoración y reparación de daños personales.

Después de obtener una casuística representativa, en un futuro esta guía podría servir para que diferentes equipos de valoradores trasladaran sus resultados al método cualitativo utilizando también métodos cuantitativos de traducción cualitativa de este daño (analíticos y formulas matemáticas que deberían ser escogidos) para proceder después al intercambio de casos y confrontación entre grupos de criterios de calificación al objeto de obtener parámetros de referencia consensuados.

Por estos motivos concluimos, calificando a ésta guía, como una "guía abierta" a la consideración de todos e inclusión en un futuro de las cuestiones que se consideren útiles. Animamos a su desarrollo y a la existencia de más colaboradores como coautores. 


\section{Guía para la valoración médico-legal de la alteración estética: daño estético/deformidad}

CLAVE n ${ }^{\circ}$ :

Fecha del examen: / /

\section{A. IDENTIFICACIÓN DEL PERITO}

Nombre:

\section{B. SOLICITANTE}

Examen solicitado por

\section{DATOS PERSONALES LESIONADO}

Nombre:

Sexo: $\square$ Masc. $\square$ Fem. Fecha de nacimiento: / /

Nacionalidad:

Dirección:

Provincia:

País:

Constitución: pesa:

C. 2 VIDA FAMILIAR

Estado civil: $\square$ casado

Hijos: $\square 1 \quad \square$

Convivientes: $\square 1$
Documento de Identidad:

Localidad:

Código Postal:

Teléfono n. ${ }^{\circ}$ :

altura: $\quad \mathrm{cm}$

C.3 VIDA ESCOLAR/FORMACIÓN:

Escolarización: $\square$ Educación infantil $\square$ Educación primaria

$\square$ Educación Secundaria Obligatoria

$\square$ Bachillerato $\quad \square$ Formación profesional ¿Cuál/es?

$\square$ Formación universitaria: ¿Cuál/es?

\section{4 VIDA LABORAL:}

Profesión actual:

Historia Laboral (trabajos anteriores):

\section{C.5 VIDA DE OCIO Y TIEMPO LIBRE}

Práctica de actividades deportivas: $\square$ no $\quad \square$ si ¿Cual?

Actividades del tiempo libre, ocio: 


\section{HECHO TRAUMÁTICO}

D.1 Fuente de los datos: $\square$ Parte lesiones $\square$ Atestado policial

D.2 Fecha del evento traumático: / /

D.3 Naturaleza del hecho traumático:

$\square$ Tráfico

$\square$ Laboral: $\square$ In itínere $\quad \square$ Lugar y tiempo de trabajo: Actividad laboral Otras: ¿Cómo?

Actividad ocio, deportiva u otras: ¿Cuál?

D.4 Otros factores causales:

D.5 Historia del hecho traumático referida por el lesionado:

\section{E. LESIONES:}

E.1 Lesiones referidas en documentos aportados:

E.2 Secuelas referidas en documentos aportados:

Pruebas diagnósticas realizadas:

Tratamientos médico/quirúrgicos realizados:

E.3 Fecha de la alta médica: / / Tratamiento actual:

\section{F. VALORACIÓN MÉDICO-LEGAL}

\section{Consolidación médico legal:}

F.1 Evolución del lesionado

$\square$ Pendiente de tratamiento con finalidad curativa:

$\square$ Curado

Secuelas

Consolidación médico-legal

- Fecha de consolidación médico-legal: / /

- Periodo: días

\section{Consecuencias lesivas hecho traumático}

F.2 Lesiones derivadas del hecho traumático:

F.3 Secuelas derivadas del hecho traumático:

F.4 Otras causas que han concurrido en la producción de las lesiones/secuelas:

- Estado Anterior patológico:

- Otras concausas concomitantes o posteriores:

\section{F.5 Incapacidad Temporal:}

- Funcional: - $\square$ Total (“encamado, ingresado"/pérdida de la autonomía) - $\square$ Parcial

- Laboral: cuales

Total

Parcial 
- Actividades del tiempo libre, lúdicas, deportivas: cuáles

$\square$ Total

$\square$ Parcial

- Afectivo-familiar: $-\square$ cuidado hijos, $\square$ padres,... - $\square$ pareja

- Escolar/formación

Vida autónoma $/ 3^{\mathrm{a}}$ persona ABVD:

$\square$ Ayuda total

$\square$ Ayuda parcial (cuantas horas al día; cuantas personas)

$\square$ Tipo de ayuda (realización actividad, estimulación, vigilancia)

$\square$ Persona cualificada, cual

$\square$ no cualificada

F.6 Incapacidad permanente:

- Funcional: Inca. Func. Permanente

Funciones afectadas, de forma total o parcial

Cuantificación:

- Laboral:

- Extralaborales:

- Escolar/Formación: $\square$ Parcial $\quad \square$ Total $\square$ Absoluta

- Tiempo libre $\square$ Parcial $\square$ Total $\square$ Absoluta

- Afectivo-familiar: $\square$ Parcial $\square$ Total $\square$ Absoluta

- Vida autónoma (ABVD):

- Desplazamiento:

- Higiene:

- Vestido:

- Alimentación:

- Determinadas actividades (AIVD): ¿Cuál?

$-\mathrm{n}^{\circ}$ de personas/tipo:

1- horas/día días/semana

2- horas/día días/semana

3- horas/día días/semana

$-\square$ Ayuda total

- $\square$ Ayuda parcial (cuantas horas al día; cuantas personas)

- $\square$ Tipo de ayuda (realización actividad, estimulación, vigilancia)

- $\square$ Persona cualificada, cual

$-\square$ no cualificada

\section{Sufrimientos padecidos, Quantum doloris}

- Temporal:

- Permanente:

Actuaciones sanitarias que implican un gasto económico:

- Temporal (conocidas, realizadas):

- Permanente (futuras): 


\section{Valoracion descriptiva del daño estético:}

\section{G.1 Secuelas:}

- Alteraciones estáticas (anatómica):

- Alteraciones dinámicas/funcional:

G.2 Elementos objetivos del daño estético (Fotografías):

a) Estado Anterior:

b) Estado actual: (fotografía)

Nota: Documento de consentimiento informado adjunto

c) Criterios de objetivación:

\section{Cicatriz (s):}

- Forma:
$\square$ puntiforme
$\square$ lineal
compleja

- Dirección/orientación:

$\square$ hay relación con las líneas de tensión cutáneas

vertical

$\square$ horizontal $\quad \square$ oblícua

- Color o pigmentación:

$\square$ normocrómica; $\square$ hipocrómica o $\quad \square$ hipercrómica

- Relieve:

$\square$ plana $\quad \square$ deprimida/profunda o $\quad \square$ elevada

- Viciosa:

$\square$ hipertrófica $\quad \square$ queloide $\quad \square$ retráctil

$\square$ con adherencias

- Frecuencia/tamaño: $\square 1 \quad \square 2 \quad \square 3 \quad \square$ múltiples

- Dimensión: 1- $\quad \mathrm{cm}$ longitudinal $-\quad \mathrm{cm}$ horizontal $-\quad \mathrm{cm}$ altura o profundidad

2- $\quad$ cm longitudinal - $\quad \mathrm{cm}$ horizontal $-\quad \mathrm{cm}$ altura o profundidad

3- cm longitudinal - $\quad \mathrm{cm}$ horizontal - $\quad \mathrm{cm}$ altura o profundidad

- Criterio topográfico:

Cara: región orbitaria, nariz, labios

Cara: partes laterales de la frente, orejas, mentón, región submentoniana

Cuello, tercio superior del tórax y manos (cabeza)

Extremidades

$\square$ Tórax, abdomen y espalda visibles ocasionalmente (deportes, playa)

$\square$ Zonas solamente visibles en caso de desnudez como nalgas y pubis

$\square$ Zonas raramente visibles o no visibles: plantas de los pies y la superficie de la cabeza oculta por el pelo

- Grado de visibilidad o distancia a la que se percibe

Distancia de las relaciones íntimas: $50 \mathrm{~cm}$.

Distancia de las relaciones sociales: $3 \mathrm{~m}$.

d) Repercusión en las actividades de la vida diaria:

- Afectiva-familiar/sexual:

- Formación/ escolar:

- Actividades profesionales:

- Actividades de ocio, deportiva:

- Repercusión sexual: 


\section{Referências}

Cobo JA. ¿Falta o delito? Algunas reflexiones médico forenses sobre las lesiones. La Ley Penal 2004 Dec.; 1(11):18-42.

Cobo JA. La valoración del daños a la personas por accidentes de tráfico. Barcelona: Bosch 2010; 5:635-706.

Criado Del Río MT. Valoración médico-legal del daño a la persona. Tomo II. Doctrina médico-legal de valoración de daños personales. Madrid: COLEX; 2010. cap.9, p.375-432.

Criado Del Río MT. Valoración médico-legal del daño a la persona. Tomo III. Doctrina médico-legal de valoración de daños personales. Madrid: COLEX; 2010. cap.1, p.25-131.

Vieira DNP, Quintero JA. Aspectos práticos da avaliação do dano corporal em Direito Civil. Coimbra: Caixa Seguros 2008; 173-226.

Resumo: Proposta de um guia para valoração médico-legal do prejuízo estético: dano estético/ deformidade

0 objetivo desse trabalho é contribuir com um "guia aberto" para a valoração médico-legal do prejuízo estético nos distintos âmbitos judiciais, cuja aplicabilidade e desenvolvimento só se demonstrarão com a experiência médico-legal e jurídica de todos os interessados na valoração e reparação dos danos pessoais. Após obter uma casuística representativa, no futuro este guia poderá servir para que diferentes equipes de peritos convertam os seus resultados num método qualitativo utilizando também métodos quantitativos de tradução qualitativa deste dano (analíticos e fórmulas matemáticas que deveriam ser seleccionadas) para proceder depois ao intercâmbio de casos e confronto entre grupos de critérios de qualificação com o objetivo de alcançar parâmetros de referência consensuais.

Palavras-chave: Prejuízo estético; dano estético; deformidade.

Summary: Proposal for a guide to medical assessment of the aesthetic alterations: aesthetic damage/deformity

This article proposes to contribute with a "open guide" for medical assessment of the aesthetic alterations in the distinct judicial fields, whose applicability and development only will be shown through the forensic and juridical experience of all the involved in the assessment and repair of personal damages. After obtaining a representative number of cases, in the future this guide can be used for different teams of professionals to translate their results into a qualitative method using also quantitative methods of qualitative translation of this damage (analytical and mathematic formulas that might to be chosen) to proceed afterwards to the exchange of cases and discussion between groups of the criteria of qualification in order to obtain consensual reference parameters.

Key-words: Aesthetic alterations; aesthetic damage; deformities. 
Résumé: Proposition d'une guide pour l'évaluation médico-légale du préjudice esthétique: dommage esthétique/difformité

Le but du travail est apporter une "guide ouverte" pour l'évaluation médico-légale du préjudice esthétique dans les divers milieux judiciaires, dont l'applicabilité et développement seulement peuvent être démontrés par l'expérience médico-légale et juridique de tous les intéressés à l'évaluation et réparation des dommages personnels. Après un nombre de cas représentatif, dans l'avenir cette guide pourrait servir pour que différentes équipes de professionnels puissent exposer leurs résultats par une méthode qualitative en utilisant aussi méthodes quantitatives de traduction qualitative de ce dommage (analytiques et des formules mathématiques que devraient être choisis) pour procéder après à l'échange d'information et confrontation parmi des groupes de critères de qualification à fin d'obtenir paramètres de référence convenables.

Mots-clés: Préjudice esthétique; dommage esthétique; difformités.

\section{Pedido de separatas:}

FERNANDA BOUCHARDET

fernanda@performo.com.br 